\title{
Practical Kramers-Kronig phase retrieval FIR filter with the Gibbs phenomenon
}

Liu, Yuyang; Li, Yan; Song, Jingwei; Luo, Ming; He, Zhixue; Yang, Chao; Lv, Jianxin; Kong, Deming; Qiu, Jifang; Hong, Xiaobin

Total number of authors:

12

Published in:

Journal of Lightwave Technology

Link to article, DOI:

10.1109/JLT.2021.3129059

Publication date:

2022

Document Version

Peer reviewed version

Link back to DTU Orbit

Citation $(A P A)$ :

Liu, Y., Li, Y., Song, J., Luo, M., He, Z., Yang, C., Lv, J., Kong, D., Qiu, J., Hong, X., Guo, H., \& Wu, J. (2022).

Practical Kramers-Kronig phase retrieval FIR filter with the Gibbs phenomenon. Journal of Lightwave

Technology, 40(4), [1007-1017]. https://doi.org/10.1109/JLT.2021.3129059

\section{General rights}

Copyright and moral rights for the publications made accessible in the public portal are retained by the authors and/or other copyright owners and it is a condition of accessing publications that users recognise and abide by the legal requirements associated with these rights.

- Users may download and print one copy of any publication from the public portal for the purpose of private study or research.

- You may not further distribute the material or use it for any profit-making activity or commercial gain

- You may freely distribute the URL identifying the publication in the public portal 


\title{
Practical Kramers-Kronig phase retrieval FIR filter with the Gibbs phenomenon
}

\author{
Yuyang Liu, Yan Li, Jingwei Song, Ming Luo, Zhixue He, Chao Yang, Jianxin Lv, Deming Kong, \\ Jifang Qiu, Xiaobin Hong, Member, IEEE, Hongxiang Guo, and Jian Wu, Member, IEEE
}

\begin{abstract}
Kramers-Kronig (KK) receiver shows strong feasibility since the phase can be extracted from the detected intensity through the Hilbert transform (HT). To reduce the complexity and make the HT more suitable for the practical KK system, the HT can be approximated as the finite impulse response (FIR) filter, i.e. the HT-FIR filter. However, the finite Fourier series expansion of the HT-FIR filter suffers from oscillations around the discontinuities and further results in an unsatisfactory inband flatness, which is the so-called Gibbs phenomenon. In the experiment and the simulation, we found that the performance of the KK system using the HT-FIR filter is strongly impaired due to the Gibbs phenomenon, by the roll-off factor of the shaping filter, frequency gap between the direct-current (DC) component and one edge of the signal spectrum, carrier-to-signal power ratio (CSPR), tap number of the HT-FIR filter, and the digital upsampling rate. We have systematically investigated the practical KK phase retrieval performance and the computational complexity considering the Gibbs phenomenon due to the adoption of the HT-FIR filter, rather than the HT used in the previous works without the Gibbs phenomenon. We conclude that there is an optimal upsampling rate when the HT-FIR filter is used, and the optimal upsampling rate is closely related to the five aspects mentioned above. We have carried out a transmission experiment using a 112-Gbit/s 16-QAM system over 1920-km with a Raman fiber amplifier to investigate the limit transmission performance of the practical KK receiver. The experimental results show that by using the 9-tap HT-FIR filter with a digital upsampling rate of 84-GHz (3-sps), the signals can be transmitted the same distance as the HT, up to $1440-\mathrm{km}$ with a bit error ratio (BER) below the $20 \%$ soft-decision forward error correction threshold.
\end{abstract}

Index Terms-Hilbert transforms, finite impulse response filters, frequency response, spectral analysis, phase retrieval, Kramers-Kronig optical receiver, optical fiber communication, digital signal processing, direct detection, single-sideband.

Yuyang Liu, Yan Li, Jingwei Song, Jifang Qiu, Yong Zuo, Xiaobin Hong, Hongxiang Guo, and Jian Wu are with State Key Laboratory of Information Photonics and Optical Communications, Beijing University of Posts and Telecommunications, Beijing, 100876, China. (e-mail: yuyangliu@bupt.edu.cn, liyan1980@bupt.edu.cn, songjingwei@bupt.edu.cn, jifangqiu@bupt.edu.cn, xbhong@bupt.edu.cn, hxguo@bupt.edu.cn, jianwu@bupt.edu.cn).

\section{INTRODUCTION}

$\mathrm{D}$ IRECT DETECTION (DD) has been an attractive receiving technology in short and medium-reach transmission systems due to its easy implementation, low cost, and low complexity [1]-[4]. However, traditional DD systems suffer from signal-signal beat interference (SSBI) and a limited transmission distance since the phase information of the optical signal is discarded during the intensity detection [5], [6].

Kramers-Kronig (KK) receiver can eliminate SSBI theoretically and enables complex full-field reconstruction as long as the minimum phase condition (MPC) is satisfied [7][10]. Compared with the traditional DD systems, the KK receiver generally has the following three advantages: (i) Fullfield reconstruction enables the use of the advanced digital signal processing (DSP) algorithms like electrical dispersion compensation (EDC); (ii) High spectral efficiency (SE) close to the coherent detection system owing to the optical-field signaling (OFS) single-sideband (SSB) modulation, and (iii) lower receiver-side complexity due to the single photodiode (PD) reception method [11]-[15].

In this DD reception method, the phase of the data-carrying signals can be uniquely extracted from the detected intensity through the Hilbert transform (HT), but an extra digital upsampling operation is essential before that [12]. Generally, an upsampling factor of at least 4 samples per symbol (4-sps) is necessary to obtain a better performance but at the expense of large computational complexity. In the practical KK phase retrieval, the HT can be approximated as the finite impulse response (FIR) filter to reduce the computational complexity, i.e. the HT-FIR filter [16]. Moreover, upsampling-free KK receiver has also been proposed to further reduce the complexity, but the adoption of two HT-FIR filters also brings additional performance deterioration, especially when the tap number of the HT-FIR filter is low [12], [17]. Thus, the performance of the HT-FIR filter under lower digital upsampling rate is quite important. Furthermore, the HT-FIR filter enabled KK receiver has shown a potential feasible in

Ming Luo, Zhixue He and Chao Yang are with Wuhan research institute of post and telecommunications, Wuhan, Hubei, 430074, China. (e-mail: mluo@wri.com.cn, zxhe@wri.com.cn, yangchao@wri.com.cn).

Jianxin Lv is with FiberHome Telecommunication Technologies Co., Ltd., Wuhan, Hubei, 430074, China. (e-mail: 1jx70@ fiberhome.com)

Deming Kong is with Department of Photonics Engineering, Technical University of Denmark, Kongens, 2800 Lyngby, Denmark (e-mail: dmkon@fotonik.dtu.dk). 


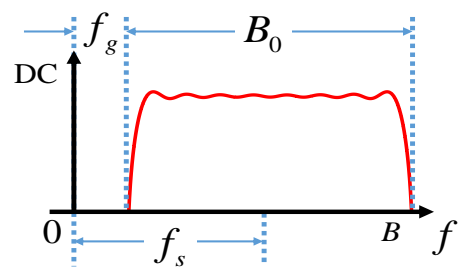

Fig. 1. The spectrum of the transmitted minimum phase signal.

practical transmission implementation and become a focus of the detailed investigation, like parallelized $\mathrm{KK}$ receiver, graphics processing unit (GPU)-based real-time KK reception, and all analogy KK system, etc. [18]-[21]. Table I shows the typical KK experiments considering different HT schemes with their tap number, SE, transmission distance, and upsampling factor. Generally, the finite Fourier series expansion of discontinuous function suffers from oscillations around the discontinuities and further results in an unsatisfactory in-band flatness, which is the so-called Gibbs phenomenon [22]-[24]. The in-band flatness of the HT-FIR filter with a larger tap number is better than that with a smaller tap number but sacrifices the computational complexity [17]. The Gibbs phenomenon has been a great challenge and widely elaborated in the DSP system, and which could usually be observed, measured, and quantified by spectrum analysis [25]-[27].

In KK receiver, the performance of phase retrieval using HT-FIR filter with the Gibbs phenomenon is mainly related to five aspects: frequency gap between the DC component and one edge of the signal spectrum $\left(f_{g}\right)$, the roll-off factor of the root raised cosine (RRC) filter, CSPR, receiver-side digital upsampling rate, and the tap number of the HT-FIR filter. These five aspects are also closely related to the advantages of the KK receiver. Firstly, the frequency gap and the roll-off factor of the RRC filter $(\alpha)$ are related to the bandwidth of the signal $(B)$ as Fig. 1 shows, and when the information bit rate is constant, the spectral efficiency will change with different frequency gap and the roll-off factor as Table. I shows. Secondly, a higher digital upsampling rate and CSPR mean a higher computational complexity and power consumption [17], [28], [29]. Thirdly, the tap number of the HT-FIR filter determines the level of the Gibbs phenomenon, which is directly related to the performance of $\mathrm{KK}$ phase retrieval. However, in the $\mathrm{KK}$ receiver, most of the previous work is based on the HT without considering the Gibbs phenomenon [13]. Although the HT-FIR filter is adopted in a few previous works, its impact on the KK phase retrieval performance and the corresponding parameters mentioned above have not been fully investigated yet [16].

In this paper, for the first time, we systematically investigate the practical KK phase retrieval performance considering the Gibbs phenomenon due to the adoption of the HT-FIR filter, rather than the HT used in the previously published works without the Gibbs phenomenon. Most of the previous work based on the HT without considering the Gibbs phenomenon reveals that the higher the digital upsampling rate, the better the KK phase retrieval performance will be [13], [28]. However, this paper provides a novel conclusion, there is an optimal upsampling rate when the HT-FIR filter is used, and the optimal upsampling rate is related to the tap number of the HT-FIR filter, CSPR, frequency gap, and the RRC roll-off factor in the KK system. Generally, the lower the tap number of the HT-FIR filter, the higher the CSPR value, the lower the frequency gap leads to a lower optimal upsampling rate. Moreover, we provide the detailed hardware complexity, mathematical derivation and analyze the spectrum change induced by the Gibbs phenomenon considering the related parameters on the KK phase retrieval performance when the HT-FIR filter is employed. In section III, a 112-Gbit/s SSB 16-QAM transmission system using KK reception over 1920-km standard single-mode fiber (SSMF) based on the Raman fiber amplifier (RFA) link is employed to investigate the limit transmission performance of the practical KK receiver. The experimental results show that the KK receiver using a 9-tap HT-FIR filter with a digital upsampling rate of $84-\mathrm{GHz}$ (3-sps) can transmit the same distance as the HT, up to $1440-\mathrm{km}$, when SD-FEC threshold of $2 \times 10^{-2}$ is considered and the complexity of the HT-FIR filter is much lower than the HT. We provide a detailed theoretical and experimental basis for the future practical implementation of the KK receiver.

\section{THEORETICAL ANALYSIS OF THE GIBBS PHENOMENON IN KK PHASE RETRIEVAL}

\section{A. The HT-FIR filter and the Gibbs phenomenon}

The Hilbert transform (HT) can be treated as a transformed system with a time domain (TD) impulse response of $h(t)$ and frequency domain (FD) transfer function of $H(f)$ :

$$
\begin{gathered}
h(t)=\frac{1}{\pi t} \\
H(f)=-j \bullet \operatorname{sign}(f)
\end{gathered}
$$

TABLE I

\begin{tabular}{|c|c|c|c|c|c|c|c|c|}
\hline Format & Symbol rate & $\begin{array}{l}\text { Hilbert } \\
\text { scheme }\end{array}$ & $\begin{array}{c}\text { Tap } \\
\text { number }\end{array}$ & Spectral efficiency & $\alpha$ & Distance & sps & Reference \\
\hline QPSK & 8-GBaud & HT & $\mathrm{LF}$ & $1.27 \mathrm{bit} / \mathrm{s} / \mathrm{Hz}$ & 0.05 & $80-\mathrm{km}$ & NM & {$[30]$} \\
\hline 16-QAM & 80-GBaud & HT-FIR & 9 to 129 & $3.64 \mathrm{bit} / \mathrm{s} / \mathrm{Hz}$ & 0.1 & 75 to $300-\mathrm{km}$ & $\mathrm{NM}$ & {$[16]$} \\
\hline 16-QAM & 40-GBaud & HT & LF & $3.44 \mathrm{bit} / \mathrm{s} / \mathrm{Hz}$ & 0.01 & $80-\mathrm{km}$ & 4 & {$[31]$} \\
\hline 16-QAM & 64-GBaud & HT & $\mathrm{LF}$ & $3.84 \mathrm{bit} / \mathrm{s} / \mathrm{Hz}$ & 0.05 & 200/1200-km & 6 & {$[32],[33]$} \\
\hline 16-QAM & 28-GBaud & HT-FIR & 16 to 64 & $3.31 \mathrm{bit} / \mathrm{s} / \mathrm{Hz}$ & 0.2 & $1440-\mathrm{km}$ & 4 & [17], [34] (previous work) \\
\hline 16-QAM & 28-GBaud & HT-FIR & 9 & $3.31 \mathrm{bit} / \mathrm{s} / \mathrm{Hz}$ & 0.2 & $1440-\mathrm{km}$ & 3 & this work \\
\hline 16-QAM & 28-GBaud & HT & $\mathrm{LF}$ & $2.83 \mathrm{bit} / \mathrm{s} / \mathrm{Hz}$ & 0.4 & $960-\mathrm{km}$ & 6 & [35] \\
\hline 16/64-QAM & 28-GBaud & HT & $\mathrm{LF}$ & $3.2 / 4.8 \mathrm{bit} / \mathrm{s} / \mathrm{Hz}$ & 0.01 & $80-\mathrm{km}$ & 2 to 7 & [36] \\
\hline 128/256-QAM & 20-GBaud & HT & LF & $6.9 / 7.88 \mathrm{bit} / \mathrm{s} / \mathrm{Hz}$ & 0.01 & $20-\mathrm{km}$ & NM & [37] \\
\hline
\end{tabular}

THE HT SCHEMES WITH THEIR TAP NUMBER, SPECTRAL EFFICIENCY, DISTANCE, AND UPSAMPLING FACTOR IN TYPICAL KK EXPERIMENTS

sps: sampling per symbol used for upsampling in KK receiver. NM: Not mentioned. LF: long frame [17]. 
where $h(t)$ and $H(f)$ are a Fourier transform pair. In practice, the HT can be approximated as an FIR filter, and its implementation in the $\mathrm{KK}$ receiver has been discussed in detail [16], [17]. Considering the problem of the time domain singularity and the periodic spectral repetition of the sampled HT's impulse response, a brick wall filter with an FD transfer function of $G_{T}(f)$ and the bandwidth corresponding to the digital upsampling rate of $R_{U}$ can be implemented in the HT. Thus, the modified HT can be calculated as the following equation shows:

$$
H_{m}(f)=-j \cdot \operatorname{sign}(f) \cdot G_{T}(f)=-j \operatorname{sign}(f),-\frac{R_{U}}{2} \leq f \leq \frac{R_{U}}{2}
$$

Then, the modified $N_{f}$-tap HT-FIR filter $\left(N_{f}\right.$ is the tap number of the HT-FIR filter) based on the FD sampling approach can be acquired by discretizing the Eq. 3 [27]:

$$
H_{m}[n]=\left\{\begin{array}{cc}
j, & 1 \leq n<N_{f} / 2 \\
-j, & -N_{f} / 2<n<0 \\
0, & n \in\left\{0,-N_{f} / 2\right\}
\end{array}\right.
$$

Afterward, the time domain impulse response of the HTFIR filter can be acquired as follows:

$$
h_{m}[n]=I D F T\left\{H_{m}[n]\right\}
$$

where $I D F T(\cdot)$ is the inverse discrete Fourier transform (IDFT) The HT-FIR filter with an odd tap number is generally used and known as the Type III filter, in which half of the tap coefficients are zero proved in [38]. Finally, the phase of the signals can be reconstructed by the convolution operation:

$$
\begin{aligned}
\varphi[k] & =\frac{1}{2} \ln \{I[n]\} * h_{m}[n]=\frac{1}{2} \ln \{I[n]\} * I D F T\{H[n]\} \\
& =\frac{1}{2} \sum_{n=\max \{0, k-L+1\}}^{\min \{L-1, k} \ln \{I[n]\} \bullet h_{m}[k-n]
\end{aligned}
$$

where $k$ represents the time index in mathematical operation and $L$ is the length of the resampled signals [17]. Fig. 2 (a) and (b) show the TD impulse response and FD transfer function of the HT, the 9-tap HT-FIR filter, and the 31-tap HT-FIR filter.
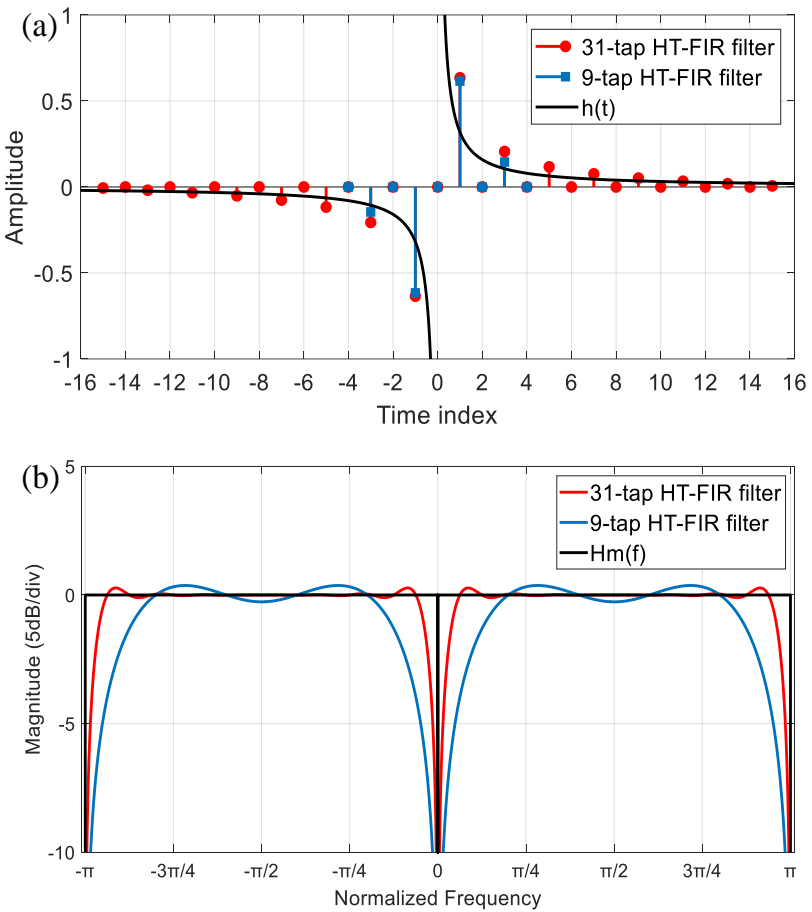

Fig. 2. (a) Time domain impulse response and (b) frequency domain transfer function of the HT, 9-tap HT-FIR filter and 31-tap HT-FIR filter.
Generally, the finite Fourier series expansion of the HT-FIR filter suffers from oscillations around the discontinuities and further results in an unsatisfactory in-band flatness, which is the so-called Gibbs phenomenon and we have briefly introduced in our previous work [17]. The in-band flatness of the HT-FIR filter with a larger tap number is better than that with a smaller tap number but sacrifices the computational complexity [12].

\section{B. Computational complexity of the HT schemes}

Based on the above principle of the HT and the HT-FIR filter, we introduce the complexity of the $\mathrm{KK}$ phase retrieval. Assuming the analog sampling rate of the analog-to-digital converter (ADC, $R_{A D C}$ ) is larger than twice the signal bandwidth, the symbol rate is $R_{s}$, and the digital upsampling is $R_{U}$ (also can be normalized to samples per symbol, sps), the oversampling factor $M=R_{U} / R_{A D C}=s p s \cdot R_{S} / R_{A D C}$ [17]. The frame length after $\mathrm{ADC}(L)$ is determined by the clock frequency $\left(R_{c l k}\right)$ and the ADC sampling rate, $L=R_{A D C} / R_{c l k}$, the length of the upsampled signal is thus $M L$ [12]. Some mathematical operations such as logarithm and root functions can be easily realized by the lookup table (LUT). The implementation of the HT-based KK phase retrieval is first to perform the FFT operation and then the FD multiplication is employed followed by the IFFT operation. Hence, the complexity of the HT-based KK phase retrieval can be represented as the number of multipliers:

$$
N_{m(F D-H T)}=2 M L \cdot \log _{2} M L+M L
$$

We then normalized the complexity to each sample:

$$
N_{m(F D-H T / \text { sample })}=2 M \log _{2} M L+M
$$

The implementation of the HT-FIR filter-based KK phase retrieval is achieved by convolution operation and the complexity is calculated as follows:

$$
N_{m(H T-F I R)}=M L\left(N_{f}-1\right) / 2
$$

It should be noted that since half of the tap coefficient of the HT-FIR filter is 0 , the actual number of the real-value multiplier is $\left(N_{f}-1\right) / 2$. The normalized complexity to each sample is:

$$
N_{m(H T-F I R / \text { sample })}=M\left(N_{f}-1\right) / 2
$$

Fig. 3 shows the computational complexity per sample of HT and the HT-FIR filter. Obviously, the complexity of the HTFIR filter-based KK phase retrieval is lower than that of the HT. Moreover, if the block-wise operation is used in parallelized or
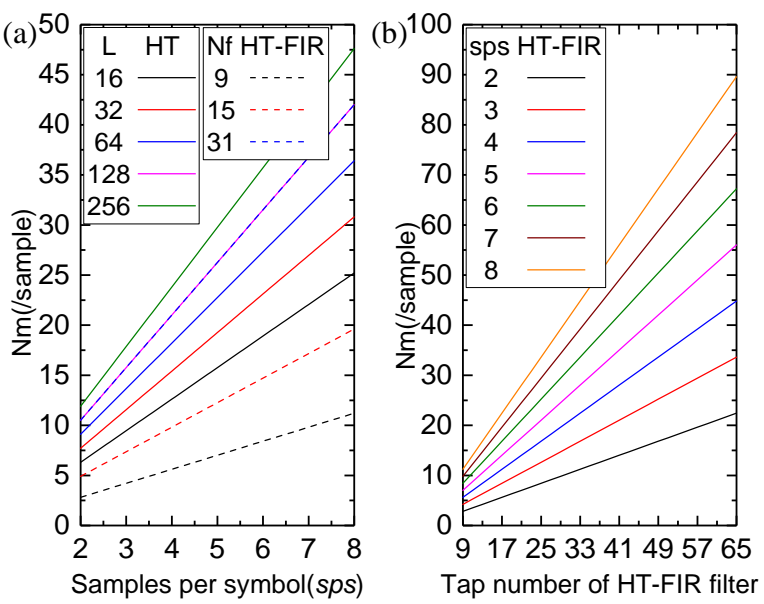

Fig. 3. The computational complexity per sample versus (a) samples per symbol (sps) and frame length $L$ of HT scheme, 9/15/31-tap HT-FIR is used a reference. (b) tap number of HT-FIR filter with different $s p s$. 
real-time KK receiver, the overlap approach (OLA) is adopted considering the edge effect, $L$ should be extended to $L+2 K$, and $K$ is the OLA length [17]. Besides, a lower digital upsampling rate implies a shorter frame length, a lower upsampling rate, and a lower demand for the total multiplier and more suitable for the cost-effective KK receiver. Most of the previous KK experiments based on the long frame (LF) operation and HT without the Gibbs phenomenon show that the higher the digital upsampling rate, the better the phase retrieval performance will be [13], [28]. However, when the HT-FIR filter with the Gibbs phenomenon is implemented, especially the number of taps is lower like the 9-tap HT-FIR filter, the results will be different.

Given the fact that the adoption of the HT-FIR filter will affect the performance of KK phase retrieval, we will then investigate the impact of the transmitter roll-factor, frequency gap, CSPR, and the receiver side digital upsampling rate considering the Gibbs phenomenon through the 28-GBaud 16QAM back-to-back (B2B) numerical simulation validation and a pseudo-random bit sequence (PRBS) with a length of $2^{14}-1$ is used for the 16-QAM symbol generation. To isolate the impact of the Gibbs phenomenon from other distortions like chromatic dispersion (CD) and the fiber nonlinearity induced by the fiber transmission, while making the impact of the Gibbs phenomenon more obvious, the system noise is only dominated by amplified spontaneous emission (ASE) noise with a simple additive white Gaussian noise (AWGN) model. Hence, the transmission simulation will not be performed to make this section of the paper focus more on the physical mechanism aspects. Then, only RRC matched filter is used rather than the multi-modulus algorithm (MMA) used for channel equalization in receiver-side DSP. Besides, the commonly used digital upsampling rate is 2 to 8 times the symbol rate and in our system, and the symbol rate is 28 -GBaud, leading to the digital upsampling rate ranges from $56-\mathrm{GHz}$ (2-sps) to $224-\mathrm{GHz}(8-$ sps). Finally, BER is evaluated using 10 million samples by point-to-point comparison.

C. Tap number of the HT-FIR filter and the digital upsampling rate considering the Gibbs phenomenon

At first, the digital upsampling rate of KK phase retrieval with different tap number of the HT-FIR filter $\left(N_{f}\right)$ is elaborated. Fig.

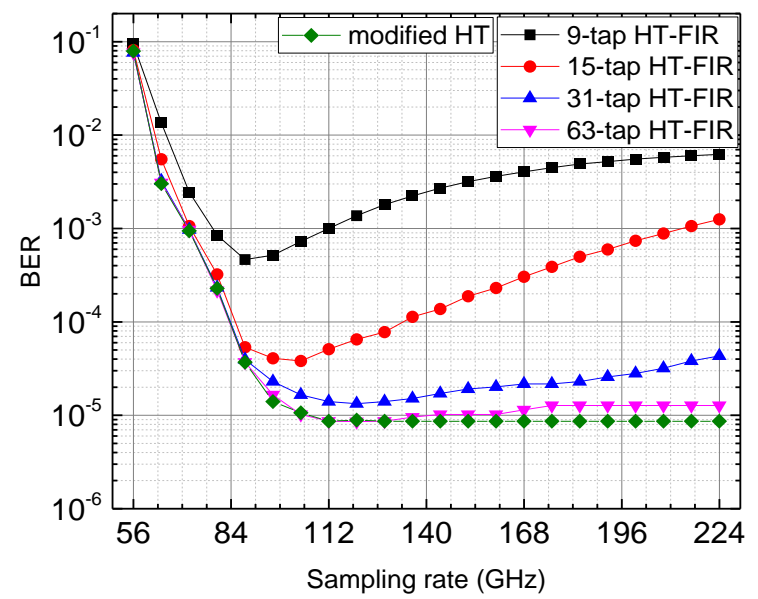

Fig. 4. BER performance versus digital upsampling rate when different tap number of the HT-FIR filter is used, modified HT is used as a reference.

4 shows the BER performance versus digital upsampling rate and different $N_{f}$ when OSNR is $28 \mathrm{~dB}$, CSPR is $11 \mathrm{~dB}$ with a frequency gap of $0.2-\mathrm{GHz}$ and a 0.2 roll-off factor, modified HT is used as a reference. It can be observed that the optimal digital upsampling rate is around $84-\mathrm{GHz}(3-\mathrm{sps})$ and $112-\mathrm{GHz}$ (4-sps) for 9 and 31-tap HT-FIR filter, respectively.

Fig. 5 shows the electrical spectrums of the recovered phase when digital upsampling rate (SR) are 56-GHz, 84-GHz with 9tap HT-FIR filter, and 224-GHz with 9/31-tap HT-FIR filter, modified HT is used as a reference. The right panel of Fig. 5 shows the corresponding constellations of the recovered complex signal. Since some nonlinear functions are operated in KK receiver, a higher upsampling rate is necessary for phase retrieval. Therefore, when a digital upsampling rate of $56-\mathrm{GHz}$ (2-sps) is used, strong spectrum aliasing is occurred resulting in a poor BER performance in all HT schemes [28]. Further increasing the upsampling rate will obtain an optimal BER, and then the BER performance begins to deteriorate. Although increasing the upsampling rate will make the spectrum component more abundant, the low-frequency part of the signals will suffer more deterioration caused by the Gibbs phenomenon of the 9-tap HT-FIR filter as shown in Fig. 5(c) and (d), Fig. 5(d) is the partial enlargement of Fig. 5(c), and the distribution of the constellation points is less dense. However,
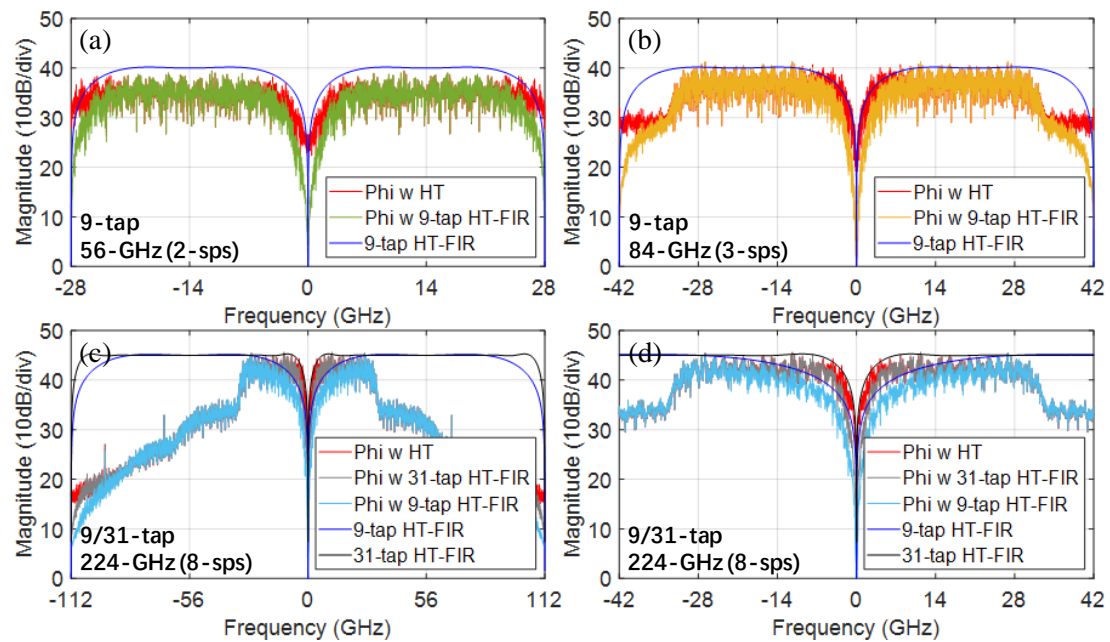

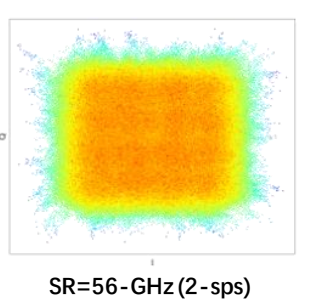

9-tap HT-FIR

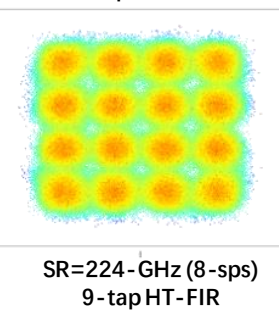

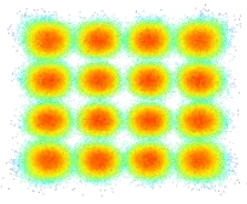

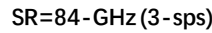

9-tap HT-FIR

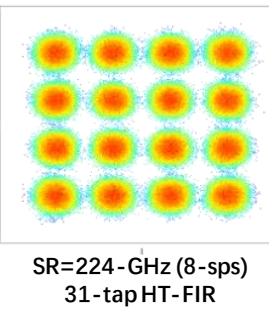

Fig. 5. Electrical spectrums of the recovered phase with a digital upsampling rate of (a) 56-GHz (2-sps), (b)84-GHz (3-sps) when 9-tap HT-FIR filter is used, (c) 224-GHz (8-sps) when 9/31-tap HT-FIR filter is used, (d) partial enlargement of (c). The blue and black lines are the FD transfer function of 9-tap and 31-tap HTFIR filters, respectively. SR: upsampling rate. The right panel shows the corresponding constellations of the recovered signal. 
when the 31-tap HT-FIR filter is adopted with an upsampling rate of 224-GHz (8-sps), the low-frequency part of the signals is hardly deteriorated compared with the 9-tap HT-FIR filter, and the distribution of the constellation will be denser.

Moreover, for the different $N_{f}$, the optimal upsampling rate is also different. When the HT-FIR filter with a lower tap number towards the lower receiver complexity is used, a lower upsampling rate is used to counter the low-frequency part distortions due to the low-pass transition gets less steep of the HT-FIR filter. However, when the complexity is not considered like a 63-tap HT-FIR filter is employed, the in-band flatness of such filter tends to be perfect and the BER performance hardly changes with a digital upsampling rate up to 224-GHz (8-sps). Generally, the larger the tap number of the HT-FIR filter, the higher the optimal upsampling rate will be, but the complexity and power consumption are quite higher and not suitable for the cost-effective system like the KK receiver. Thus, the limited tap number of the HT-FIR filter can be used to achieve a trade-off between the receiver complexity and the BER performance.

\section{CSPR and the digital upsampling rate considering the Gibbs phenomenon}

We then investigate the impact of the Gibbs phenomenon among different CSPR values. Fig. 6 shows the BER performance versus digital upsampling rate when different CSPR values and tap number of the HT-FIR filters are considered, OSNR is $28 \mathrm{~dB}$ with a frequency gap of $0.2-\mathrm{GHz}$ and a 0.2 roll-off factor resulting in a signal bandwidth $(B)$ of 33.8-GHz, modified HT is used as a reference. It can be observed that when the CSPR increases, the optimal upsampling rate will be decreased.

Then, Eq. 11 shows the Taylor expansion of the detected signals after the logarithm operation, and the simple PD model is considered without the additional noise:

$$
\begin{aligned}
& \ln \sqrt{I(t)}=\frac{1}{2} \ln [I(t)]=\frac{1}{2} \ln |A+s(t)|^{2}=\frac{1}{2} \ln \left\{[A+s(t)]\left[A+s^{*}(t)\right]\right\} \\
& =\ln A+\underbrace{\frac{1}{2}\left[\frac{s(t)}{A}+\frac{s^{*}(t)}{A}\right]}_{\text {first-order }}+\underbrace{\frac{1}{2}\left[\frac{s^{2}(t)}{2 A^{2}}+\frac{s^{* 2}(t)}{2 A^{2}}\right]}_{\text {second-order }}+\underbrace{\frac{1}{2}\left[\frac{s^{3}(t)}{3 A^{3}}+\frac{s^{* 3}(t)}{3 A^{3}}\right]}_{\text {third-order }}+\cdots
\end{aligned}
$$

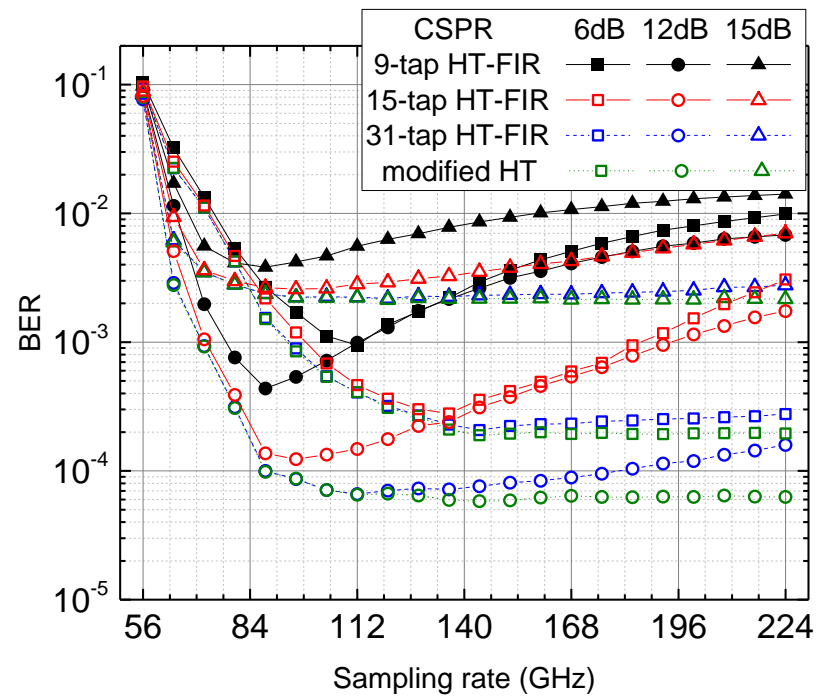

Fig. 6. BER performance versus digital upsampling rate when CSPR are 6, 12, and $15 \mathrm{~dB}$, as well as different tap number of HT-FIR filters are considered, modified HT is used as a reference.

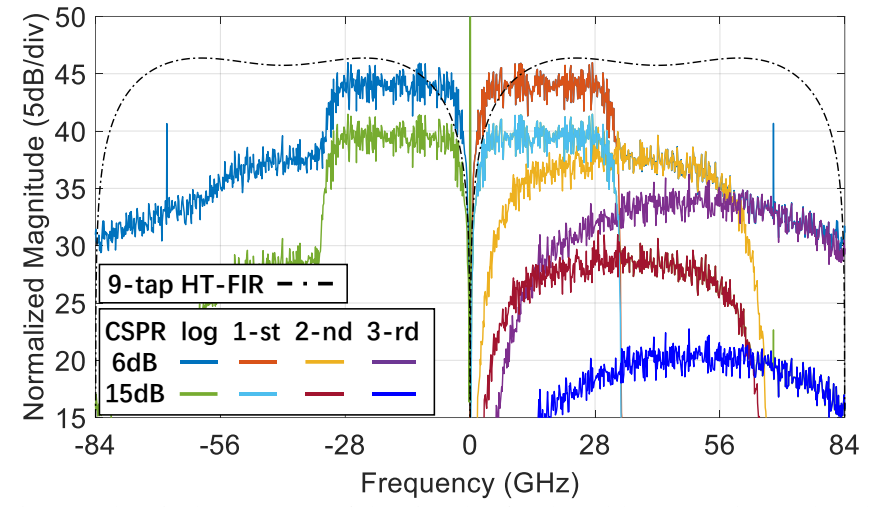

Fig. 7. Electrical spectrums of the signals after logarithm operation and each order terms when $\mathrm{CSPR}=6 \mathrm{~dB}$ and $15 \mathrm{~dB}$ with a upsampling rate of $168-\mathrm{GHz}(6-$ $s p s)$. The black dotted line is the FD transfer function of the 9-tap HT-FIR filter.

where $s^{*}(t)$ is the conjugation of the original signal $s(t)$ and $A$ is the DC component. Appendix shows the detailed derivation and FFT of Eq. 11.

Since increasing the CSPR means increasing $A$ in Eq. 11, the power of the signals after the logarithm (log-signal) operation will be concentrated more on the low-order terms, especially the first order term. Fig. 7 shows the electrical spectrums of each order component and after the logarithm (log) operation when CSPR $=6 \mathrm{~dB}$ and $15 \mathrm{~dB}$ with a digital upsampling rate of $168-\mathrm{GHz}$ (6-sps). It should be noted that the upsampling rate of $168-\mathrm{GHz}$ is not the optimal value, and we plot it to show the spectrum of different order terms. Since the positive and the negative frequency parts of the electrical spectrums are symmetrical, we only plot the first, second, and third order Taylor expansion spectrums on the positive frequency part. It can be easily observed that when $C S P R=15 \mathrm{~dB}$, the power difference of different order terms will be more obvious, and the power of the log-signals is concentrated more on the first order term compared with $\mathrm{CSPR}=6 \mathrm{~dB}$.

Thus, when a lower tap HT-FIR filter is used for phase retrieval, like the 9-tap HT-FIR filter. As mentioned above, when the log-signal with a higher CSPR is considered, like $\mathrm{CSPR}=15 \mathrm{~dB}$, the power will be concentrated more on the first order term, a lower digital upsampling rate is necessary to counter the low-frequency part distortions due to the low-pass transition gets less steep of the HT-FIR filter. Besides, when the $\log$-signal with a lower CSPR is considered, like CSPR $=6 \mathrm{~dB}$, the power is more dispersed and a relative higher upsampling rate is essential to obtain more abundant spectrum components while keep better high-frequency and low-frequency performance at the same time. It should be noted that the performance gap between different CSPR is due to the linear and nonlinear penalties when minimum phase condition is satisfied [28]. We should pay more attention to the power differences caused by different CSPR as mentioned above. So, a trade-off between CSPR values (refers to power consumption) and digital upsampling rate (refers to complexity) needs to be considered to achieve a better phase retrieval performance.

\section{E. The frequency gap and the digital upsampling rate considering the Gibbs phenomenon}

We also evaluate the Gibbs phenomenon associated with the frequency gap and the transmitter shaping filter roll-off factor. Generally, RRC shaping is needed to form the signal at the 


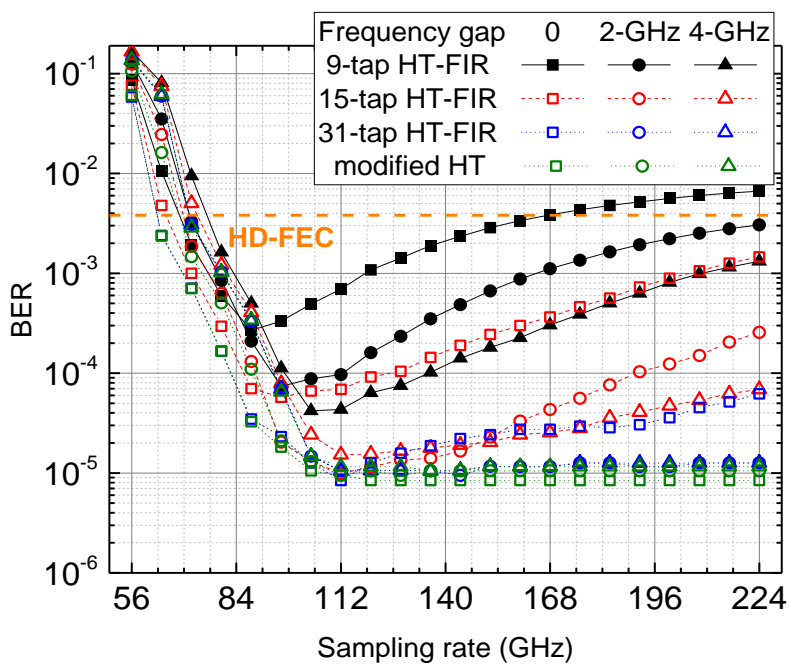

Fig. 8. BER performance versus digital upsampling rate when frequency gap is 0,2 , and 4-GHz, as well as different tap number of the HT-FIR filters are considered, modified HT is used as a reference.

transmitter to generate the minimum phase signal [8]. The rollfactor of the RRC filter and the frequency gap show a trade-off between the required OSNR and spectral efficiency [39].

Fig. 8 shows the BER performance versus digital upsampling rate and the frequency gap when different tap number of the HT-FIR filters are considered, the modified HT is used as a reference. It should be noted that considering the spectral efficiency, we only investigate the frequency gap of $0,2-\mathrm{GHz}$, and 4-GHz for the 28-GBaud 16-QAM system, CSPR is $11 \mathrm{~dB}$ and OSNR is $28 \mathrm{~dB}$. The impact of the digital upsampling rate has been illustrated in detail, generally, a larger frequency gap is adopted to avoid the impact of SSBI [13]. However, when the HT-FIR filter is adopted, the results might be different. When the digital upsampling is fixed to $84-\mathrm{GHz}$ (3-sps) and the frequency gap is 0 , the low-frequency part is impaired due to the Gibbs phenomenon resulting in a poor BER performance as Fig. 9 (a) shows. When the frequency gap is 4-GHz, although the low-frequency part is hardly affected, the high-frequency part cannot be obtained abundantly resulting in a worse BER performance as Fig. 9(b) shows. Then, when the digital upsampling rate is fixed to $112-\mathrm{GHz}$ (4-sps) and the frequency gap is 0 , although more spectrum component is obtained to improve the BER performance, the discarded low-frequency part will seriously deteriorate BER performance as shown in Fig. 9(c). However, when the frequency gap is $4-\mathrm{GHz}$, we not only obtain more spectrum components, but also the lowfrequency part is less impaired, resulting in an improved BER performance as Fig. 9(d) shows. Moreover, increasing the frequency gap is an effective approach to mitigate the SSBI, and the SSBI can be eliminated by the HT-FIR filter as Fig. 9(b) and (d) show. Besides, increasing the frequency gap will also be an approach against the Gibbs phenomenon, which prevents the low-frequency part be affected by the Gibbs phenomenon when the HT-FIR filter is used as above mentioned. Generally, a larger frequency gap means a larger corresponding optimal upsampling rate. This is related to the above mentioned, a larger frequency gap implies that the spectrum of the signals moves to the higher frequency part, and the Gibbs phenomenon generally affects the signal from the zero-frequency as Fig. 9 shows. It can be observed that there is almost no spectrum component from 0 to $4-\mathrm{GHz}$ (frequency gap of 4-GHz), so it effectively reduces the impact of the Gibbs phenomenon on the lowfrequency part of the signals. Then, increasing the digital upsampling rate appropriately to obtain more abundant highfrequency components, and acquire a better KK phase retrieval performance. Therefore, a trade-off between the frequency gap (refers to the spectral efficiency) and the digital upsampling rate (refers to computational complexity) is also necessary for KK phase retrieval.

\section{F. The transmitter roll-off factor and the digital upsampling rate considering the Gibbs phenomenon}

Finally, the impact of the transmitter roll-off factor is elaborated. The base bandwidth of the data-carrying signals is $14.14-\mathrm{GHz}$, 14.7-GHz, and 16.8-GHz, respectively, when the roll-off
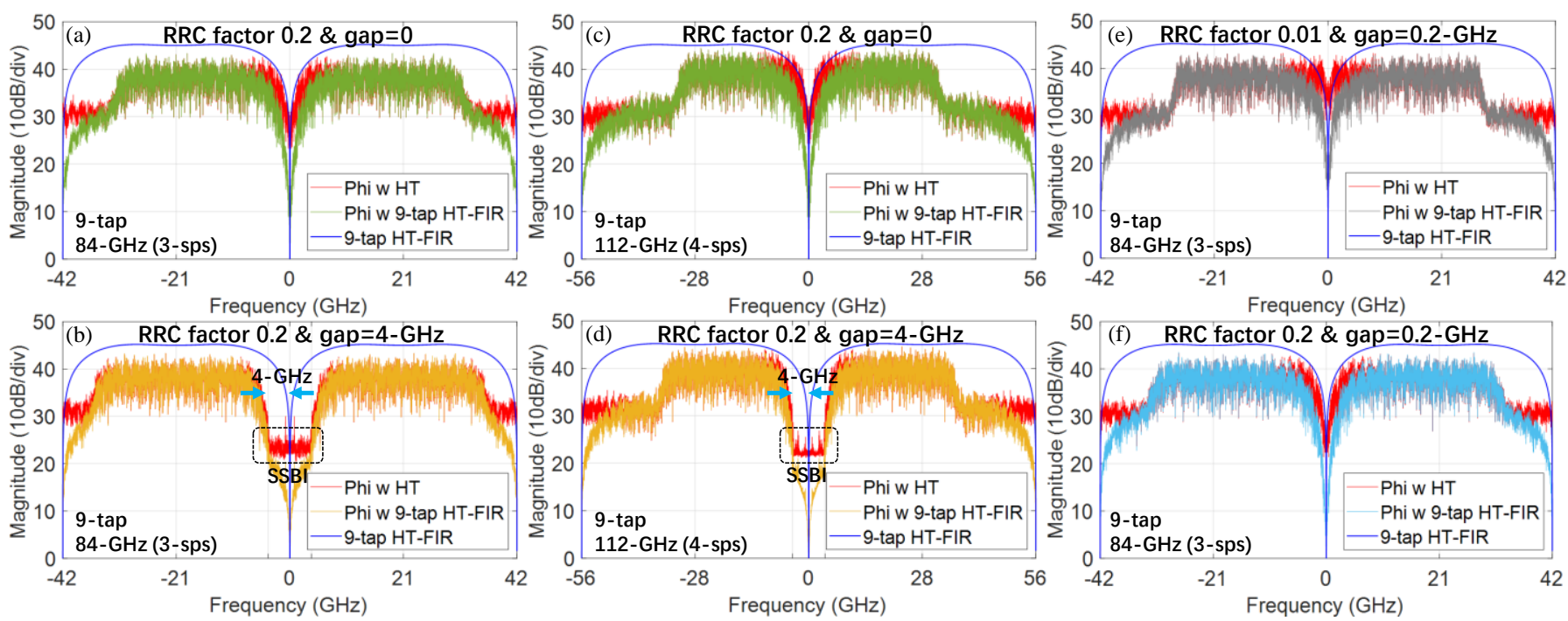

Fig. 9. Electrical spectrums of the recovered phase with (a) 0.2 roll-off factor of RRC filter and 0 frequency gap when SR is $84-\mathrm{GHz}$ (3-sps); (b) 0.2 roll-off factor of RRC filter and 4-GHz frequency gap when SR is 84-GHz (3-sps); (c) 0.2 roll-off factor of RRC filter and 0 frequency gap when SR is 112-GHz (4-sps); (d) a 0.2 roll-off factor of RRC filter and 4-GHz frequency gap when SR is 112-GHz (4-sps); (e) a 0.01 roll-off factor of RRC filter and 0.2-GHz frequency gap when $\mathrm{SR}$ is $84-\mathrm{GHz}$ (3-sps); (f) a 0.2 roll-off factor of RRC filter and 0.2-GHz frequency gap when SR is 84-GHz (3-sps). SR: upsampling rate. 


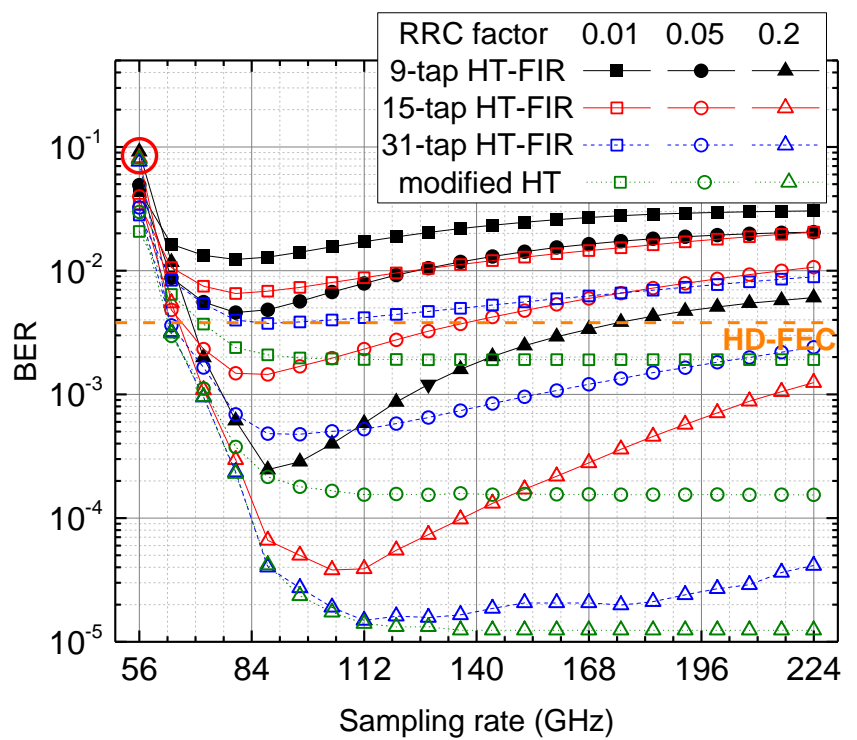

Fig. 10. BER performance versus digital upsampling rate when RRC roll-factor are $0.01,0.05$, and 0.2 , as well as different tap number of HT-FIR filters are considered, modified HT is used as a reference.

factors of $0.01,0.05$, and 0.2. Fig. 10 shows the BER performance versus different digital upsampling rate and RRC roll-off factors when OSNR is 28dB and 9/15/31-tap HT-FIR filter is adopted, modified HT is used as a reference. Generally, the roll-off factor of the RRC filter is positive to the bandwidth, a larger roll-off factor implies that the low-pass transition of the transmitted signal will be slower, and the received signal after logarithm operation will suffer fewer distortions caused by the Gibbs phenomenon and vice versa as Fig. 9(e) and (f) show. Fig 9(e) and (f) show the electrical spectrums of the log-signal when the roll-off factor is 0.01 and 0.2 , the digital upsampling rate is $84-\mathrm{GHz}$ (3-sps). Obviously, the signal with a 0.01 roll-off factor suffers more low-frequency distortions than that of the 0.2 rolloff factor. Besides, the increased bandwidth of the signal means that the bandwidth of different order parts after logarithm operation will be increased, a higher digital upsampling rate is essential to obtain more abundant spectrum components. However, even if the signal with a larger roll-off factor has a higher demand for the digital upsampling rate, the larger the roll-off factor, the better the phase retrieval performance, and the larger the optimal upsampling rate will be [16].

\section{G. Summary of the Gibbs phenomenon}

In summary, the performance of the HT-FIR filter-based KK phase retrieval considering the Gibbs phenomenon can be summarized as follows:

(i) the tap number of the HT-FIR filter will determine the in-band flatness of the FIR filter, that is, the degree of the Gibbs phenomenon. The larger the tap number of the HT-FIR filter, the better the KK phase retrieval performance will be. Generally, a lower tap number of the HT-FIR filter has poor inband flatness and ripple, but lower complexity, and vice versa. When the HT-FIR filter is employed, there is an optimal upsampling rate for the KK phase retrieval performance, and the optimal upsampling rate is generally related to the CSPR, the frequency gap, and the roll-off factor of the RRC filter.

(ii) The digital upsampling rate is related to the computational complexity, which also determines how many signals can be included in the band. A higher upsampling rate means a more abundant spectrum component, but the lowfrequency and high-frequency parts of the signal after the logarithm operation will be worse when the HT-FIR filter is used. Therefore, the upsampling rate can be reduced appropriately to obtain better performance of the low-frequency part of the signal, and vice versa. Besides, parameters related to bandwidth and signal energy concentration, such as CSPR, frequency gap, and RRC roll-off factor, will also determine the optimal upsampling rate.

(iii) CSPR is related to the power consumption and the minimum phase condition, which also determines the signal power concentration. Generally, a larger CSPR implies that the signals are concentrated more on the first order term, so a better in-band flatness at the low-frequency part is more significant to obtain a better KK phase retrieval performance and vice versa. Thus, a larger CSPR has a lower optimal upsampling rate.

(iv) The frequency gap is related to the spectral efficiency, which determines the position of the signals that move to the high-frequency part. Increasing the frequency gap can relax the low-frequency part distortion due to the Gibbs phenomenon, but at the expense of the spectral efficiency. Generally, a lower upsampling rate implies a high-frequency discarded due to the larger frequency gap, thus, a higher upsampling rate is essential to obtain more abundant spectrum information. Therefore, a higher frequency gap receives a better $\mathrm{KK}$ phase retrieval performance but the optimal upsampling rate will be higher.

(v) The roll-off factor is also related to the spectral efficiency and the signal bandwidth. A larger roll-off factor leads to a larger signal bandwidth, the bandwidth and the power of different order part of the received signal after logarithm operation will be different. Therefore, a larger roll-off factor generally has a larger optimal upsampling rate to obtain more abundant spectrum information.

We have systematically elaborated the performance of the HT-FIR filter in the practical KK system for the first time, and analyze the impact of the Gibbs phenomenon on the KK phase retrieval considering tap number, digital upsampling rate, CSPR, the roll-off factor, and the frequency gap. We conclude that when the tap number of the HT-FIR filter is lower (9-tap HT-FIR filter) to reduce the complexity, 3-sps is an effective digital upsampling rate to obtain the optimal BER performance. When the tap number of the HT-FIR filter is higher (31 or more tap HT-FIR filter) to satisfy the superior performance, 4-sps is an effective digital upsampling rate to obtain the optimal BER performance.

\section{EXPERIMENTAL SETUP AND RESULTS}

\section{A. Experimental setup}

Fig. 11 shows the experimental setup and DSP flow of the SSB DD 16-QAM KK reception system. The 28-GBaud RRC shaped 16-QAM signal with a roll-factor of 0.2 is firstly preprocessed in offline DSP. The independent I, Q paths output by 65-GSa/s arbitrary waveform generator (AWG, Keysight M8195A, 3-dB bandwidth of 25-GHz) are amplified by two 50$\mathrm{GHz}$ linear electric amplifiers (EA, SHF s807) and subsequently send to the IQ modulator (Fujitsu FTM $7961 \mathrm{EX} / 301$ ) with a 3 -dB bandwidth of 22-GHz. Then, a polarization-maintaining erbium-doped fiber amplifier (PM- 


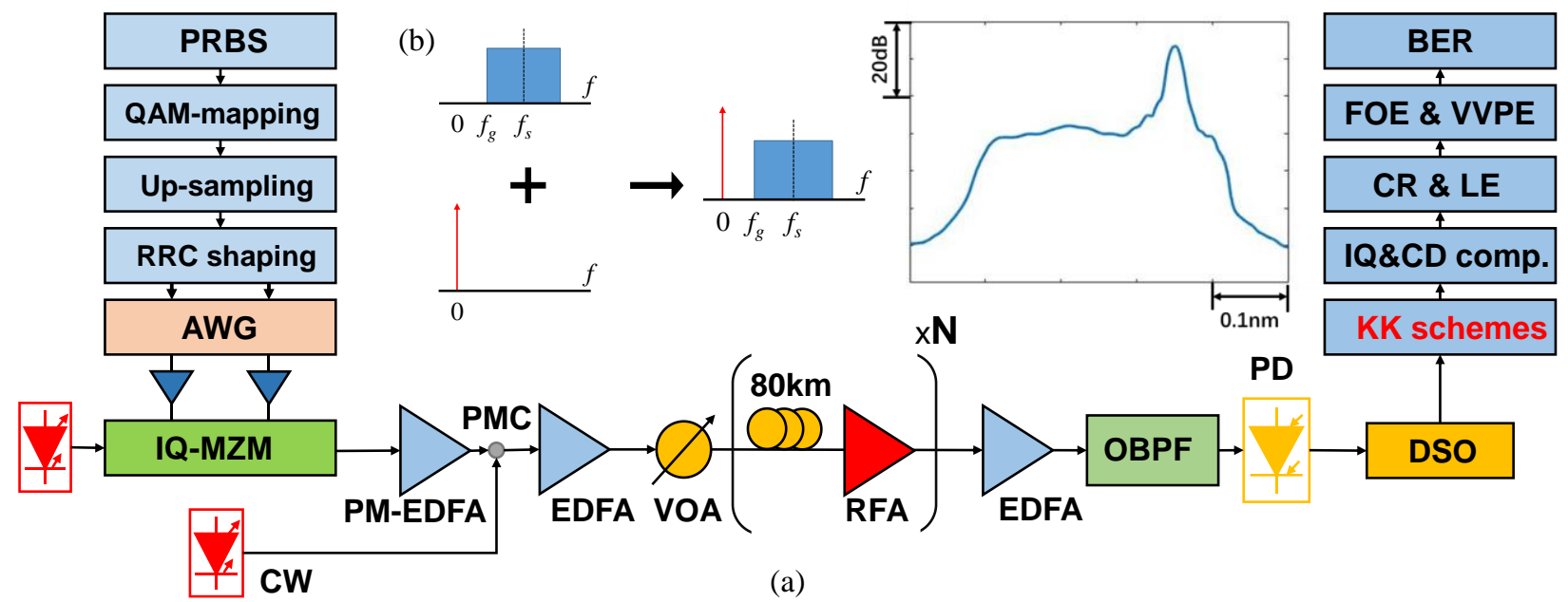

Fig. 11. (a) Experimental setup and offline DSP flow. (b) Generation of optical tone based SSB signal with its optical spectrum. PRBS: pseudo-random binary sequence; RRC: root raise cosine; AWG: arbitrary waveform generator; IQ-MZM: IQ Mach-Zehnder modulators; PMC: polarization-maintaining coupler; EDFA: Erbium-doped fiber amplifier; VOA: variable optical attenuator; RFA: Raman fiber amplifier; OBPF: optical band-pass filter; PD: photo-detector; DSO: digital sampling oscilloscope; CD: chromatic dispersion; CR: clock recovery; LE: linear equalizer.

EDFA) is used to amplify the optical signal to expand the range of CSPR adjustment and soon coupled with a continuous wave (CW) tone through a polarization-maintaining coupler (PMC). It should be noted that the optical carrier of the data-carrying signal (193.4-THz) and the CW tone (193.383-THz) are both generated using a 4-channel tunable laser source (Keysight N7714A, linewidth $<100-\mathrm{kHz}$ ), which guarantees the minimum phase condition (MPC) and the same polarization. Before the fiber transmission link, an Erbium-doped fiber amplifier (EDFA) and a variable optical attenuator (VOA) are used for optical launched power adjustment. The fiber transmission link is made up of multi-spans standard single-mode fiber (SSMF) of 80-km with Raman fiber amplifiers (RFA) to acquire a limit transmission performance. It should be noted that our fiber links are cascaded rather than loop controlled. After fiber transmission, the signal is amplified by another EDFA and then filtered by a tunable optical band-pass filter (OBPF) with a 3$\mathrm{dB}$ bandwidth of $0.4-\mathrm{nm}$ to adjust the input power of the subsequent photodetector (PD) and remove the out-of-band noise. Finally, the filtered signal is detected by an AC-coupled $\mathrm{PD}$ with a $3-\mathrm{dB}$ bandwidth of 70-GHz, sampled by an $80-\mathrm{GSa} / \mathrm{s}$ digital sampling oscilloscope (DSO, Lecroy LabMaster 10$36 \mathrm{Zi}-\mathrm{A}$ ) with the $36-\mathrm{GHz}$ bandwidth, and processed offline.

\section{B. Transmitter-side and receiver-side DSP algorithms}

For the transmitter-side, a pseudo-random bit sequence (PRBS) with a length of $2^{14}-1$ is used for the 16-QAM Gray mapping and then upsampled to 2 samples per symbol (2-sps) for the Nyquist shaping resulting in a $16.8-\mathrm{GHz}$ baseband bandwidth and a $0.2-\mathrm{GHz}$ frequency gap. For the receiver-side, the detected signals are firstly reconstructed by the KK receiver considering the different digital upsampling rate and the tap number of HT-FIR filters. Afterward, in-phase and quadrature (IQ) imbalance compensation based on the Gram-Schmidt orthogonalization procedure (GSOP) algorithm is used for possible IQ imbalance caused by the IQ modulator. Then, the frequency domain electrical dispersion compensation (FDEDC) and 5-order Bessel filter are adopted for chromatic dispersion (CD) compensation and out-of-band noise mitigation. Moreover, T/2-clock recovery (CR) based on the
Gardner algorithm and linear equalization (LE) based on the multi-modulus algorithm (MMA) with 15-tap are used in turn for data synchronization and channel equalization. Considering the phase noise caused by the laser linewidth and other optoelectrical $(\mathrm{O} / \mathrm{E})$ devices, the frequency offset estimation (FOE) based on the fast Fourier transform (FFT) and the carrier phase estimation (CPE) based on the Viterbi-Viterbi phase estimation (VVPE) algorithm with a block length of 64 are employed. Finally, after performing the extended Kalman filter (EKF) based equalization algorithm, BER is calculated using 10 million points after de-mapping and decision.

\section{Back-to-back case}

We first investigate the BER performance versus CSPR when different tap number of the HT-FIR filters are considered as Fig. 12 shows, the modified Hilbert transform (HT) is used as a reference. For all HT and the HT-FIR filters, the BER performance is improved as CSPR increases and then tends to be stable when CSPR is greater than $12 \mathrm{~dB}$. Due to the limited bandwidth of the RF cable at the transmitter and about 4-sps upsampling rate is used for KK phase retrieval, the demand for CSPR will be higher [16], [17], [40], [41]. Besides, the BER performance of $\mathrm{CSPR}=14 \mathrm{~dB}$ is little deteriorated, which is

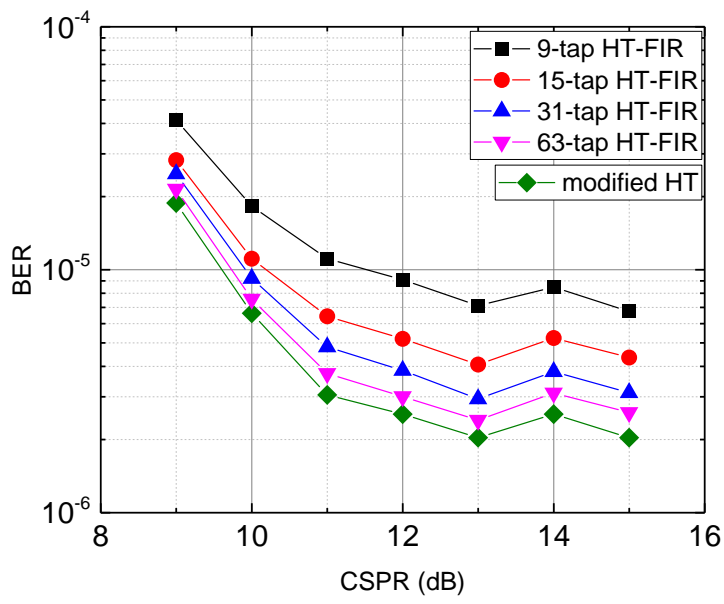

Fig. 12. BER performance versus CSPR when different tap number of HTFIR filter is considered. 


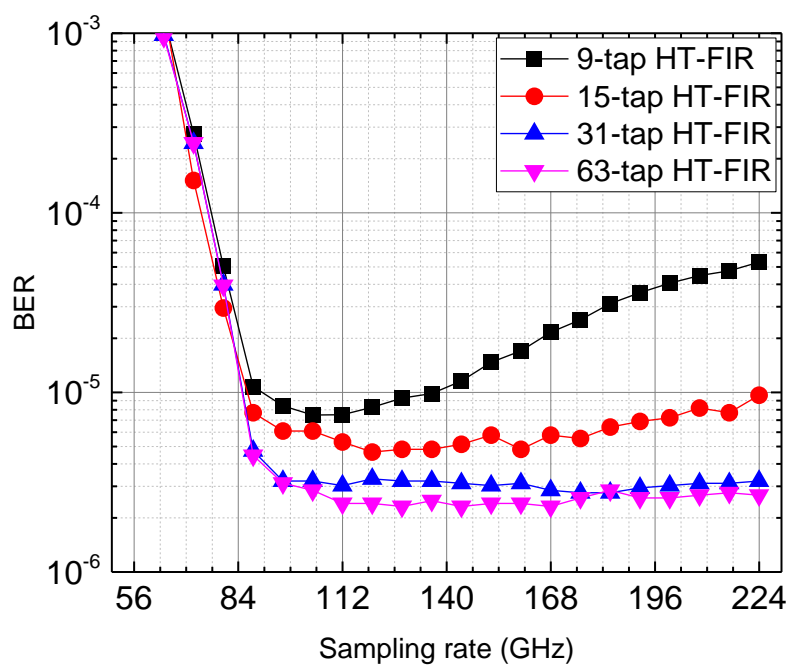

Fig. 13. BER performance versus digital upsampling rate when different tap number of HT- FIR filter is considered

caused by the modulator bias jitter, or the polarization of the DC component and the signal is not exactly the same due to the fiber disturbance, and further resulting in some power loss when the signal and the DC beat in the square-law detection [42]. As expected, the 9-tap HT-FIR filter does not work well as the Gibbs phenomenon of which is quite high even with the help of the subsequently DSP algorithms described above. However, the BER performance is almost unchanged when the tap number of the HT-FIR filter is larger than 31 and the result is the same as the numerical simulation.

Next, the BER performance versus digital upsampling rate is discussed when different tap number of the HT- FIR filters are considered as Fig. 13 shows. Since a 0.2 roll-off factor of the RRC filter is used in our experiment, the base bandwidth of the data-carrying signal is thus $16.8-\mathrm{GHz}$. Besides, the frequency shifting of $17-\mathrm{GHz}$ (i.e. frequency gap is $0.2-\mathrm{GHz}$ ) is employed resulting in a bandwidth of the final SSB signal to be 33.8-GHz see the experimental setup. The common upsampling rate from $56-\mathrm{GHz}(2-s p s)$ to $224-\mathrm{GHz}(8-s p s)$ is adopted and CSPR is kept at $13 \mathrm{~dB}$. Similar to the numerical simulation results, there is an optimal upsampling rate for the HT-FIR filter, especially when the tap number is small, like the 9-tap HT-FIR filter. However, the performance gap is not obvious as the simulation results. This is because the multi-modulus algorithm (MMA) is employed in the offline DSP, which is necessary and can effectively compensate for the channel imperfect response and the Gibbs phenomenon [43], [44]. Moreover, the BER performance begins to be stable when the upsampling rate is larger than $90-\mathrm{GHz}$ (around 3-sps, 84-GHz), this result is also similar to the previous work [16]. However, the tap number of the HT-FIR filter cannot be further reduced as the compensation ability of MMA is limited. Although the 7-tap HT-FIR filter can still recover the full field based on the KK principle, the BER performance will be seriously deteriorated. Therefore, it is not worth sacrificing such a large performance just to reduce the complexity, and the complexity per sample of the 7-tap and the 9-tap HT-FIR differs by only $M$ multipliers. Thus, the 9-tap HTFIR filter is the optimal choice considering the complexity and the performance in the practical KK implementation.

\section{Fiber transmission case}

Fiber transmission is carried out using a Raman fiber amplifier (RFA) link with different fiber lengths. CSPR and optical launch power have a great impact on the KK transmission performance. CSPR is associated with the MPC while OSNR is related the fiber nonlinearity [17]. Thus, the joint optimization of CSPR and the optical launch power at $960-\mathrm{km}$ and $1440-\mathrm{km}$ are shown in Fig. 14(a) and (b). Besides, the 9-tap and the 31tap HT-FIR filters with an upsampling rate of $84-\mathrm{GHz}$ (3-sps) are considered in fiber transmission. For $960-\mathrm{km}$ fiber transmission, the optimal launch power is 5,6 , and $7 \mathrm{dBm}$ at CSPR is 11,12 , and $13 \mathrm{~dB}$. For $1440-\mathrm{km}$ fiber transmission, the optimal launch power is 6,7 , and $8 \mathrm{dBm}$ at CSPR is 12,13 , and $14 \mathrm{~dB}$. Thus, the optimal CSPR and optical launch power are $12 \mathrm{~dB}$ and $6 \mathrm{dBm}$ for $960-\mathrm{km}$ fiber transmission as well as $13 \mathrm{~dB}$ and $7 \mathrm{dBm}$ for $1440-\mathrm{km}$ fiber transmission.

Finally, the BER performance versus different fiber lengths is performed to investigate the limit transmission performance of KK reception using the HT-FIR filter as shown in Fig. 14(c), modified HT is used as a reference. It can be observed that when the fiber transmission distance is increased, the performance gap between the HT-FIR filter with different tap number will be smaller. Moreover, the BER of the 9-tap HT-FIR filter and the 31-tap HT-FIR filter with an upsampling rate of 84-GHz (3sps) can be lower than the hard-decision forward error correction (HD-FEC) threshold of $3.8 \times 10^{-3}$ at $960-\mathrm{km}$ fiber transmission and lower than the soft-decision forward error correction (SD-FEC) threshold of $2 \times 10^{-2}$ at $1440-\mathrm{km}$ fiber transmission, the same as the HT.
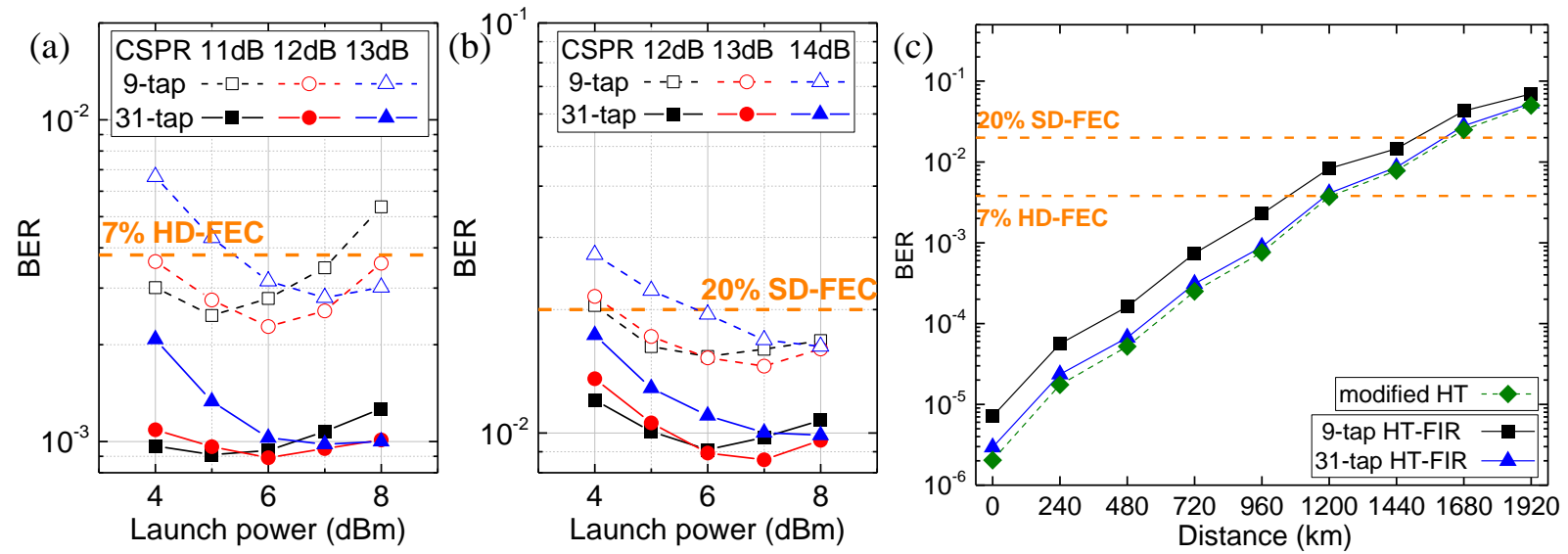

Fig. 14. BER performance versus CSPR and optical launch power: (a) with a fiber length of 960-km; (b) with a fiber length of 1440-km. (c) BER performance versus different fiber length. HD-FEC: hard-decision forward error correction of $3.8 \times 10^{-3}$; SD-FEC: soft-decision forward error correction of $2 \times 10^{-2}$. 


\section{CONCLUSIONS}

We have systematically investigated the practical KK phase retrieval performance considering the Gibbs phenomenon due to the adoption of the HT-FIR filter, rather than the Hilbert transform (HT) used in the previously published works without the Gibbs phenomenon. Most of the previous work reveals that the higher the digital upsampling rate, the better the KK phase retrieval performance will be. However, we provide a novel conclusion, there is an optimal upsampling rate when the HTFIR filter is used for KK phase retrieval. We performed a detailed analysis on the optimal digital upsampling rate in the HT-FIR filter-based KK receiver and its interplay with system parameters: CSPR, frequency gap, and RRC roll-off factor in numerical simulation. We conclude that when the tap number of the HT-FIR filter is lower (9-tap HT-FIR filter) to reduce the complexity, 3-sps is an effective digital upsampling rate to obtain the optimal BER performance. When the tap number of the HT-FIR filter is higher (31 or more tap HT-FIR filter) to satisfy the superior performance, 4-sps is an effective digital upsampling rate to obtain the optimal BER performance.

We have also experimentally demonstrated the performance of the HT-FIR filter in a 112-Gbit/s single polarization (SP) 16QAM direct detection (DD) transmission system over 1920-km SSMF using KK reception. The back-to-back results show that the 31-tap HT-FIR filter has a similar performance to that of the HT. Moreover, fiber transmission experiment is carried out using a Raman fiber amplifier (RFA) link, and the results show that the BER performance of the 9-tap HT-FIR filter-based KK reception with an upsampling rate of $84-\mathrm{GHz}$ (3-sps) can be lower than the HD-FEC threshold of $3.8 \times 10^{-3}$ after $960-\mathrm{km}$ fiber transmission and lower than the SD-FEC threshold of $2 \times 10^{-2}$ after $1440-\mathrm{km}$ fiber transmission. We provide a detailed theoretical and experimental basis for the future practical implementation of the KK receiver.

\section{APPENDIX}

We now derive Eq. 11 by using Taylor expansion:

$$
\begin{aligned}
\ln \sqrt{I(t)} & =\frac{1}{2} \ln [I(t)]=\frac{1}{2} \ln |A+s(t)|^{2}=\frac{1}{2} \ln \left\{[A+s(t)]\left[A+s^{*}(t)\right]\right\} \\
& =\frac{1}{2}\left\{\ln [A+s(t)]+\ln \left[A+s^{*}(t)\right]\right\} \\
& =\ln A+\frac{1}{2}\left\{\ln \left[1+\frac{s(t)}{A}\right]+\ln \left[1+\frac{s^{*}(t)}{A}\right]\right\} \\
& =\ln A+\frac{1}{2}\left\{\sum_{n=1}^{\infty} \frac{[s(t) / A]^{n}}{n}+\sum_{n=1}^{\infty} \frac{\left[s^{*}(t) / A\right]^{n}}{n}\right\} \\
& =\ln A+\underbrace{\frac{1}{2}\left[\frac{s(t)}{A}+\frac{s^{*}(t)}{A}\right]}_{\text {first-order }}+\underbrace{\frac{1}{2}\left[\frac{s^{2}(t)}{2 A^{2}}+\frac{s^{* 2}(t)}{2 A^{2}}\right]}_{\text {second-order }} \\
& +\underbrace{\frac{1}{2}\left[\frac{s^{3}(t)}{3 A^{3}}+\frac{s^{* 3}(t)}{3 A^{3}}\right]}_{\text {third-order }}+\cdots
\end{aligned}
$$

where $s^{*}(t)$ is the conjunction operation. The FFT of Eq. 12 is:

$$
\begin{aligned}
& F[\ln \sqrt{I(t)}]=\ln A \cdot \delta(f)+\underbrace{\frac{1}{2}\left\{\frac{S(f)}{A}+\frac{[S(-f)]^{*}}{A}\right\}}_{\text {first-order }}+ \\
& \underbrace{\frac{1}{2}\left\{\frac{S(f) * S(f)}{2 A^{2}}+\frac{[S(-f)]^{*} *[S(-f)]^{*}}{2 A^{2}}\right\}}_{\text {second-order }}+ \\
& \underbrace{\frac{1}{2}\left\{\frac{S(f) * S(f) * S(f)}{3 A^{3}}+\frac{[S(-f)]^{*} *[S(-f)]^{*} *[S(-f)]^{*}}{3 A^{3}}\right\}}_{\text {third -order }}+\cdots
\end{aligned}
$$

where $*$ is the convolution operation.

\section{REFERENCES}

[1] K. Zhong, X. Zhou, Y. Wang, T. Gui, Y. Yang, J. Yuan, L. Wang, W. Chen, H. Zhang, J. Man, L. Zeng, C. Yu, A. Lau, and C. Lu, "Recent advances in short reach systems," in Proc. of Opt. Fiber Commun. Conf., 2018, paper Tu2D.7.

[2] K. Zhong, X. Zhou, J. Huo, C. Yu, C. Lu and A. Lau, "Digital signal processing for short reach optical communications: A review of current technologies and future trends," J. Lightw. Technol., vol. 36, no.2, pp. 377-400, 2018.

[3] P. J. Winzer, D. T. Neilson, and A. R. Chraplyvy, "Fiber-optic Transmission and Networking: The Previous 20 and the Next 20 Years [Invited]," Opt. Express, vol. 26, no. 18, pp. 24190-24239, 2018.

[4] G. N. Liu, L. Zhang, T. Zuo and Q. Zhang, "IM/DD Transmission Techniques for Emerging 5G Fronthaul, DCI, and Metro Applications," J. Lightw. Technol., vol. 36, no. 2, pp. 560-567, 2018.

[5] C. Li, M Luo and X. Li, “4 × 128-Gb/s PDM-DMT signal transmission over 1440-km SSMF with high phase noise tolerance," Opt. Express, vol. 26, no. 23, pp. 30901-30910, 2018.

[6] X. Chen, C. Antonelli, S. Chandrasekhar, G. Raybon, A. Mecozzi, M. Shtaif, and P. J. Winzer, "Kramers-Kronig receivers for 100-km datacenter interconnects," J. Lightw. Technol., vol. 36, no. 1, pp. 79-89, 2018.

[7] A. Mecozzi, C. Antonelli, and M. Shtaif, "Kramers-Kronig coherent receiver," Optica, vol. 3, no. 11, pp. 1220-1227, 2016.

[8] A. Mecozzi, "A necessary and sufficient condition for minimum phase and implications for phase retrieval," 2016, arXiv: 1606.04861.

[9] A. Mecozzi, "Retrieving the full optical response from amplitude data by Hilbert transform," Opt. Commun., vol. 282, no. 20, pp. 4183-4187, 2009.

[10] T. Wang, and A. J. Lowery, "Minimum Phase Conditions in KramersKronig Optical Receivers," J. Lightw. Technol., vol. 38, no. 22, pp. 62146220, 2020.

[11] S. T. Le et al., "1.72-Tb/s Virtual-Carrier-Assisted Direct-Detection Transmission Over 200 km," J. Lightw. Technol., vol. 36, no. 6, pp. 1347-1353, 2018.

[12] T. Bo and H. Kim, "Toward practical Kramers-Kronig receiver: Resampling, performance, and implementation," J. Lightw. Technol., vol. 37, no. 2, pp. 461-469, 2019.

[13] A. Mecozzi, C. Antonelli, and M. Shtaif, "Kramers-Kronig receivers," Advances Opt. Photon., vol. 11, no. 3, pp. 480-517, 2019.

[14] Y. Liu, Y. Li, H. Zhou, Z. Zhao, L. Yue, H. Guo, J. Qiu, X. Hong, W. Li, Y. Zuo, and J. Wu, "Performance Analysis of Extended Kalman Filter in Kramers-Kronig Receiver," in Proc. of Opto. Elec. and Commun. Conf., 2019, paper ThC3.

[15] X. Chen, C. Antonelli, S. Chandrasekhar, G. Raybon, J. Sinsky, A. Mecozzi, M. Shtaif, and P. Winzer, "218-Gb/s single-wavelength, single-polarization, single-photodiode transmission over $125-\mathrm{km}$ of standard singlemode fiber using Kramers-Kronig detection," in Proc. of Opt. Fiber Commun. Conf., 2017, paper Th5B.6. 
[16] C. Fullner, M. H. Adib, S. Wolf, J. N. Kemal, W. Freude, C. Koos, S. Randel, "Complexity Analysis of the Kramers-Kronig Receiver," J. Lightw. Technol., vol. 37, no. 17, pp. 4295-4307, 2019.

[17] Y. Liu, Y. Li, J. Song, L. Yue, H. Zhou, M. Luo, Z. He, J. Qiu, Y. Zuo, W. Li, X. Hong, H. Guo and J. Wu, "Transmission of a 112-Gbit/s 16QAM over a 1440-km SSMF with parallel Kramers-Kronig receivers enabled by an overlap approach and bandwidth compensation," Opt. Express, vol. 29, no. 6, pp. 8117-8129, 2021.

[18] S. van der Heide et al., "Real-time, Software-Defined, GPU-Based Receiver Field Trial," in Proc. of European Conf. on Optical Commun., 2020, pp. 1-4.

[19] S. van der Heide et al., "Field Trial of a Flexible Real-Time SoftwareDefined GPU-Based Optical Receiver," J. Lightw. Technol., vol. 39, no. 8, pp. 2358-2367, 2021.

[20] A. J. Lowery, T. Wang, and B. Corcoran, "Towards analogue KramersKronig receivers," in Proc. of European Conf. on Optical Commun., 2019, pp. 1-4.

[21] T. Harter, C. Füllner, J. N. Kemal, S. Ummethala, J. L. Steinmann, M. Brosi, J. L. Hesler, E. Bründermann, A.-S. Müller, W. Freude, S. Randel1 and C. Koos, "Generalized Kramers-Kronig receiver for coherent terahertz communications," Nature Photonics, vol. 14, no. 10, pp. 1-6, 2020.

[22] D. Gottlieb, C. W. Shu, A. Solomonoff and H. Vandeven, "On the Gibbs phenomenon I: Recovering exponential accuracy from the Fourier partial sum of a non-periodic analytic function", J. Comput. Appl. Math., vol. 43, no. 1, pp. 81-98, Nov. 1991

[23] D. Gottlieb and C.-W. Shu, "On the Gibbs phenomenon and its resolution", SIAM Rev., vol. 39, no. 4, pp. 644-668, Dec. 1997.

[24] B. D. Shizgal and J.-H. Jung, "Towards the resolution of the Gibbs phenomena", J. Sci. Comput., vol. 161, no. 1, pp. 41-65, Dec. 2003.

[25] J. W. Gibbs, "Fourier series", Nature, vol. 606, pp. 200, Apr. 1899.

[26] A. V. Oppenheim, A. S. Willsky and S. Hamid, Signals and Systems, Upper Saddle River, NJ, USA: Prentice-Hall, 1983.

[27] J. G. Proakis, and M. Salehi, "Digital Communications, fifth edition," 2008.

[28] Z. Li et al., "Joint optimisation of resampling rate and carrier-to-signal power ratio in direct-detection Kramers-Kronig receivers," in Proc. of European Conf. on Optical Commun., 2017, Paper W.2.D.3.

[29] T. Bo, and H. Kim, "Coherent versus Kramers-Kronig Transceivers in Metro Applications: A Power Consumption Perspective," in Proc. of Opt. Fiber Commun. Conf., 2019, paper M1H.7.

[30] Q. Zhang, and C. Shu, "Kramers-Kronig Detection of Polarization Multiplexing Signals by a Single-Ended Photodiode," in Proc. Opt. Fiber Commun. Conf., 2018, Paper W2A.55.

[31] Y. Zhu, M. Jiang, and F. Zhang, "Direct detection of polarization multiplexed single sideband signals with orthogonal offset carriers," Opt. Express, vol. 26, no. 12, pp. 15887-15898, 2018.

[32] S. T. Le, K. Schuh, M. Chagnon, F. Buchali, R. Dischler, V. Aref, , F. Buchali and K. M. Engenhardt, "1.72-Tb/s virtual-carrier-assisted directdetection transmission over 200 km," J. Lightw. Technol., vol. 36, no. 6, pp. 1347-1353, 2018.

[33] S. T. Le, K. Schuh, F. Buchali, M. Chagnon, and H. Bülow, "1.6-Tb/s WDM direct detection transmission with virtual-carrier over $1200 \mathrm{~km}$," in Proc. Opt. Fiber Commun. Conf., 2018, Paper Tu2D.5.

[34] Y. Liu, Y. Li, J. Song, H. Zhou, L. Yue, X. Li, M. Luo and J. Wu, "Parallel implementation of KK receiver enabled by heading-frame architecture and bandwidth compensation," in Proc. Opt. Fiber Commun. Conf., 2020, paper M3J.5.

[35] L. Shu, J. Li, Z. Wan, Z. Yu, X. Li, M. Luo, S. Fu, and K. Xu, "Singlephotodiode 112-Gbit/s 16-QAM transmission over 960-km SSMF enabled by Kramers-Kronig detection and sparse I/Q Volterra filter," Opt. Express, vol. 26, no. 19, pp. 24564-24576, 2018.

[36] Z. Li, M. S. Erkilinc, K. Shi, E. Sillekens, L. Galdino, T. Xu, B. C. Thomsen, P. Bayvel, and R. I. Killey et al., "Spectrally efficient 168 GB/s/ $\lambda$ WDM 64-QAM single-sideband Nyquist-subcarrier modulation with Kramers-Kronig direct-detection receivers," J. Lightw. Technol., vol. 36, no. 6, pp. 1340-1346, 2018.

[37] Y. Zhou, J. Yu, Y. Wei, R. Deng, L. Zhao, N. Chi, G. Chang, and Y. Zhang, "160 Gb/s 256QAM Transmission in a $25 \mathrm{GHz}$ Grid Using Kramers-Kronig Detection," in Proc. Opt. Fiber Commun. Conf., 2019, paper Th2A.46.

[38] Y. Liu, Y. Li, J. Song, M. Luo, Z. He, C. Yang, J. Lv, J. Qiu, X. Hong, $\mathrm{H}$. Guo and $\mathrm{J}$. $\mathrm{Wu}$, "Investigation of the low-complexity Hilbert FIR filter enhanced 112-Gbit/s SSB 16-QAM transmission with parallelized Kramers-Kronig reception over 1440-km SSMF," Opt. Express, vol. 29, no. 8, pp. 38796-38810, 2021.

[39] M. S. Erk1linç et al., "Spectrally Efficient WDM Nyquist Pulse-Shaped 16-QAM Subcarrier Modulation Transmission with Direct Detection," $J$. Lightw. Technol., vol. 33, no. 15, pp. 3147-3155, 2015.

[40] X. Chen et al., "Kramers-Kronig Receivers for 100-km Datacenter Interconnects," J. Lightw. Technol., vol. 36, no. 1, pp. 79-89, 2018.

[41] X. Chen et al., "Impact of O/E Front-End Frequency Response on Kramers-Kronig Receivers and its Compensation," in Proc. of European Conf. on Optical Commun., 2018, pp. 1-3.

[42] C. Antonelli et al. "Polarization Multiplexing with the Kramers-Kronig Receiver," J. Lightw. Technol., vol. 35, no. 24, pp. 5418-5424, 2017.

[43] J. Yang, J. Werner and G. A. Dumont, "The multimodulus blind equalization and its generalized algorithms," in IEEE J. Sel. Areas Commun., vol. 20, no. 5, pp. 997-1015, 2002.

[44] E. M. Ip and J. M. Kahn, "Fiber Impairment Compensation Using Coherent Detection and Digital Signal Processing," J. Lightw. Technol., vol. 28 , no. 14 , pp. 502-519, 2010. 